\title{
Parteienwettbewerb und Wahrscheinlichkeit verschiedener Koalitionsoptionen bei der Bundestagswahl 2021
}

\author{
Marc Debus
}

Eingegangen: 7. August 2021 / Überarbeitet: 14. Oktober 2021 / Angenommen: 22. Oktober 2021 / Online publiziert: 25. November 2021

(C) Der/die Autor(en) 2021

Zusammenfassung Das komplexer gewordene bundesdeutsche Parteiensystem und die Angleichung in der Stärke der Parteien bei Wahlen führt zu einem ebenfalls komplexeren Koalitions- und Regierungsbildungsprozess auf Bundes- wie Landesebene. Diese Kurzanalyse präsentiert die programmatischen Profile der im Bundestag vertretenen Parteien auf der Grundlage ihrer zur Bundestagswahl 2021 verfassten Wahlprogramme. Im Anschluss daran wird - auf der Basis der Determinanten der Regierungs- und Koalitionsbildung in Deutschland in den Bundesländern und auf Bundesebene seit 1990 - mit Rückgriff auf unterschiedliche Szenarien der Sitzverteilung im 20. Deutschen Bundestag, die neben den sich aus dem Wahlergebnis ergebenden Parteienkonstellationen auch andere Koalitionsoptionen umfassen, die jeweils wahrscheinlichste Regierungskoalition ermittelt. Die Ergebnisse verdeutlichen, dass die Regierungsbildung in Deutschland von office- und policy-orientierten Koalitionstheorien sowie den institutionell-kontextuellen Rahmenbedingungen entscheidend beeinflusst wird und eine „Ampelkoalition“ das wahrscheinlichste Ergebnis des Regierungsbildungsprozesses 2021 ist, wenn eine ,große Koalition“ aus Union und SPD als ausgeschlossen betrachtet wird.

Schlüsselwörter Koalitionsbildung · Bundestagswahl 2021 · Deutschland · Parteienwettbewerb · Koalitionswahrscheinlichkeiten 


\title{
Party Competition and the Probability of Various Coalition Options in the 2021 Federal Election
}

\begin{abstract}
The growing complexity of the federal German party system and the alignment in the strength of the parties in recent elections leads to a more complex coalition and government formation process at the federal and state level. This contribution presents the policy profiles of the parties represented in the Bundestag on the basis of their 2021 election manifestos. Furthermore, we present - on the basis of the determinants of government formation in Germany at the state and federal level since 1990 - the most likely coalition using different scenarios of the distribution of seats in the 20th German Bundestag, including the election outcome. The findings show that government formation in Germany is determined by office- and policy-seeking coalition theories and by institutional-contextual factors. A coalition between the Social Democrats (SPD), the Greens, and the Free Democrats (FDP) is the most likely outcome of the 2021 government formation process if another "grand coalition" between the Christian Democrats and Social Democrats is considered as rejected.
\end{abstract}

Keywords Coalition formation · Bundestag election 2021 · Germany · Party competition $\cdot$ Coalition probabilities

\section{Einleitung}

Vor dem Hintergrund des langwierigen Regierungsbildungsprozesses in Deutschland nach der Bundestagswahl 2017 ist auch der Prozess der Koalitionsbildung nach der Bundestagswahl vom 26.09.2021 nicht einfacher geworden. Da Mehrheiten im Bundestag für ideologisch ,lagerkonforme“ und seitens der jeweiligen Parteien gewünschte Koalitionen aus Union (CDU/CSU) und Freien Demokraten (FDP) einerseits oder aus Sozialdemokraten (SPD) und Bündnis 90/Die Grünen andererseits unwahrscheinlich sind, ist die Bildung programmatisch heterogenerer Parteikombinationen wie einer „Ampelkoalition“ aus SPD, Grünen und FDP oder einer „Jamaikakoalition“" aus CDU/CSU, Grünen und FDP wohl unausweichlich, wenn denn nicht das Experiment einer Minderheitsregierung angestrebt werden sollte (vgl. etwa Stecker 2017).

Der Ausgang des Koalitionsbildungsprozesses hängt nicht nur von den institutionellen Rahmenbedingungen ab, sondern auch von den Strategien, die den individuellen Nutzen der Parteien maximieren (Laver und Schofield 1998; Martin und Stevenson 2001). Um ihre Ziele bestmöglich durchzusetzen, streben Parteien nach der Kontrolle über möglichst viele Ämter und nach der Implementation möglichst vieler von ihnen versprochener Politikinhalte. Zudem spielen die Koalitionsaussagen der Parteien eine Rolle, die sie im Vorfeld der Wahl getätigt haben. Um das Vertrauen der Wählerinnen und Wähler nicht zu verspielen, tun Parteien ihr Möglichstes, nicht mit solchen Parteien zu koalieren, die vor der Wahl noch als Koalitionspartner abgelehnt wurden. Gleichzeitig versuchen Parteien wiederum solche Koalitionen nach der Wahl zu bilden, die sie im Wahlkampf als wünschenswerte parteipolitische 
Zusammensetzung der Regierung genannt haben (vgl. für eine Übersicht mit Bezug auf Deutschland Bräuninger et al. 2019a, S. 83-85; Debus et al. 2021, S. 250-259).

Auch institutionelle Faktoren beeinflussen das Ergebnis des Regierungsbildungsprozesses. So sind in parlamentarischen Systemen, die durch den sogenannten positiven Parlamentarismus gekennzeichnet sind, in denen ein Premierminister oder eine Kanzlerin von einer Mehrheit im Parlament in das Amt hineingewählt werden muss, Minderheitsregierungen seltener als in Fällen, in denen das Staatsoberhaupt aufgrund der Mehrheitsverhältnisse im Parlament einen Regierungschef ernennt und sich erst bei Abstimmungen im Parlament nach der Wahl zeigt, ob der neu ernannte Premier oder Kanzler tatsächlich über eine stabile Mehrheit verfügt (Bergman 1993). Der deutsche Föderalismus setzt - über den Einfluss des Bundesrates auf die Gesetzgebung, konkret bei Zustimmungsgesetzen (Stecker 2016) - einen weiteren institutionellen Anreiz, der die Chancen für bestimmte Parteienkombinationen erhöht, die nächste Regierung zu bilden. Dies gilt für solche möglichen Koalitionen, die über eine Mehrheit in der Länderkammer verfügen. Aufgrund der Ausdifferenzierung des deutschen Parteiensystems und einer damit verbundenen großen Varianz in den Stärken der Parteien zwischen den Bundesländern ist jedoch eine Mehrheit für die die Bundesregierung tragenden Parteien zunehmend schwer zu erreichen. So verfügte etwa die "große Koalition“" aus CDU/CSU und SPD in der Legislaturperiode von 2017 bis 2021 über keine Mehrheit im Bundesrat und benötigte die Unterstützung der in zahlreichen Landesregierungen vertretenen Grünen, um für zustimmungspflichtige Gesetze im Bundesrat eine Mehrheit zu erreichen.

Der folgende Beitrag präsentiert zum einen die programmatischen Profile der im Bundestag vertretenen Parteien auf der Grundlage ihrer zur Bundestagswahl 2021 verfassten Wahlprogramme. Im Anschluss daran werden - auf der Basis der Determinanten der Regierungs- und Koalitionsbildung in Deutschland in Bund und Ländern seit 1990 - die Resultate eines bedingten logistischen Regressionsmodells interpretiert. Die Resultate dieser Schätzung werden in einem zweiten Schritt herangezogen, um auf der Grundlage unterschiedlicher Szenarien der Sitzverteilung im 20. Deutschen Bundestag die jeweils wahrscheinlichste Regierungskoalition zu ermitteln. Abschließend werden die Ergebnisse zusammengefasst, Schlussfolgerungen gezogen und offene Fragen - wie etwa die nach dem Einfluss der Koalitionswünsche der Wähler sowie innerparteilicher Konflikte auf die Regierungsbildung - diskutiert.

\section{Theoretischer Hintergrund, Daten und Methoden}

Die koalitionstheoretische Literatur identifiziert vier Faktoren und Prozesse als entscheidend für die parteipolitische Zusammensetzung einer Regierung. Diese sind (1) das Wahlergebnis bzw. die Sitzstärke der Parteien, auf die sich die an der Maximierung von politischen Ämtern ausgerichteten Koalitionstheorien konzentrieren (von Neumann und Morgenstern 1944; Riker 1962); (2) die sachpolitisch-inhaltliche Positionierung der parteipolitischen Akteure, was dem policy-orientierten Ansatz der Koalitionstheorie entspricht und wo zwischen der (parteispezifischen) Bedeutung eines Politikfelds (Salienz) und der Position einer Partei zu den entsprechenden Sachfragen unterschieden wird (vgl. Axelrod 1970; De Swaan 1973; Laver und Shepsle 
1996; Bräuninger et al. 2019a); (3) die institutionell-kontextuellen Faktoren des politischen Prozesses im Allgemeinen und des Regierens im Besonderen (vgl. Strøm et al. 1994; Bäck und Dumont 2008); (4) die seitens der Parteien mitunter geäuBerten, positiv wie negativ formulierbaren Koalitionsaussagen (Golder 2006; Debus 2009).

In Deutschland übt der Mehrebenencharakter des politischen Systems einen Einfluss auf die Regierungsbildung aus. So wirken die bundespolitischen Mehrheitsverhältnisse - in Abhängigkeit des zeitlichen Abstands zwischen Landtags- und Bundestagswahl (Däubler und Debus 2009) - auf die Regierungsbildung in den Bundesländern ein (vgl. Kropp 2001; Pappi et al. 2005). Um ihre Politikziele auch im Bundesrat durchsetzen zu können, sollten die an der Bundesregierung beteiligten Parteien versuchen, parteipolitisch gleiche Landesregierungen zu installieren, um damit das Lager der Regierungsländer im Bundesrat zu stärken. Umgekehrt ist zu erwarten, dass die Oppositionsparteien auf Bundesebene die Schwächung des Regierungslagers zum Ziel haben. Trotz der Ausdifferenzierung des bundesdeutschen Parteiensystems aufgrund der Etablierung der Linken und der AfD zeigt sich nach wie vor ein signifikanter Einfluss der parteipolitischen Zusammensetzung von Regierung und Opposition im Bundestag auf die Regierungsbildung in den Bundesländern (vgl. Bräuninger et al. 2020, S. 224-226). Gleichzeitig ist es von Vorteil für die die Bundesregierung tragenden Parteien, wenn sie über eine Mehrheit in der Länderkammer verfügen, um auf diese Weise zustimmungspflichtige Gesetze, die trotz Föderalismusreform noch immer rund $40 \%$ der Bundesgesetzgebung ausmachen (Stecker 2016), leichter durch den Bundesrat zu bringen. Nach der erfolgreichen Bildung einer „Deutschland-Koalition“ aus CDU, SPD und FDP in Sachsen-Anhalt erreichen Union, SPD und Grüne keine Mehrheit mehr im Bundesrat; eine ,Ampelkoalition" ist noch weiter von einer Mehrheit in der zweiten Kammer entfernt. Die neue Bundesregierung würde die Unterstützung der Landesregierungen benötigen, in denen neben den Unionsparteien, der SPD und den Grünen auch die FDP vertreten ist, um eine Mehrheit im Bundesrat für zustimmungspflichtige Gesetzesinitiativen zu erreichen.

Zudem kommt „kontextuellen“ Merkmalen ein entscheidender Einfluss auf die parteipolitische Zusammensetzung von Regierungen zu (vgl. Strøm et al. 1994; Martin und Stevenson 2010). So weisen Koalitionsoptionen mit Parteien, mit denen in der Vergangenheit bereits Erfahrungen im gemeinsamen Regieren gesammelt werden konnten, im Allgemeinen geringere Unsicherheiten und niedrigere Transaktionskosten auf (vgl. Bäck und Dumont 2007). Zu den Kontextmerkmalen zählt auch der Ausschluss von einer oder mehreren Parteien aus dem Koalitionsspiel aufgrund ihres Status als „Antisystempartei“. Einzelne Parteien können einerseits generell, also von allen anderen Mitbewerbern, von der Regierungsbildung a priori ausgeschlossen werden, andererseits aber auch nur von einigen Parteien als von vornherein ,nicht regierungsfähig“" deklariert werden (Debus 2009). Gleiches gilt - mit umgekehrtem Vorzeichen - für positiv formulierte Koalitionsaussagen oder Vorwahlallianzen (Bräuninger et al. 2020, S. 220-224). Solche Koalitionsaussagen oder -ablehnungen werden vielfach von den Parteivorsitzenden oder -vorständen wenige Wochen vor der Wahl öffentlich erklärt und mitunter auch von Parteitagen offiziell verabschiedet. Informationen zu den formell (von Parteitagen) oder weniger formell (etwa von 
den Vorsitzenden oder Spitzenkandidaten einer Partei) getätigten Koalitionsaussagen werden den Wahlberichten entnommen, die regelmäßig in der Zeitschrift für Parlamentsfragen erscheinen, sowie - im Falle jüngerer Wahlen - entsprechenden Medienberichten.

Um die Implikationen der programmatischen Positionierung der Parteien für die Regierungsbildung und damit die aktuellen Muster des Parteienwettbewerbs für den Regierungsbildungsprozess 2021 analysieren zu können, werden Informationen zu den Positionen der Bundesparteien auf den zentralen Politikfeldern sowie zu der parteispezifischen Bedeutung (Salienz) dieser Politikfelder benötigt. Zur Ermittlung des programmatischen Profils der Parteien auf den beiden den deutschen Parteienwettbewerb maßgeblich prägenden Konfliktdimensionen - dem Gegensatz zwischen einem „,starken“, intervenierenden und einem „schwachen“, zurückhaltenden Staat in der Wirtschafts- und Sozialpolitik einerseits und dem Gegenüber von progressiv-liberalen und traditionell-konservativen Positionen in der Innen-, Rechts- und Gesellschaftspolitik andererseits (vgl. Pappi und Shikano 2004; Bräuninger et al. 2020) - wird hier auf das rein computergestützte „Wordscores“-Verfahren zurückgegriffen (Laver et al. 2003). Zwar gibt es keinen „Goldstandard“ für die Ermittlung der programmatischen Positionen politischer Parteien unter der Fülle von entsprechenden Datenquellen wie manueller Inhaltsanalysen von Wahlprogrammen, etwa des MARPOR-Datensatzes (Volkens et al. 2017) oder von Expertenbefragungen (Benoit und Laver 2006; Bakker et al. 2015; Jankowski et al. 2021). Jedoch ergeben sich insbesondere für Deutschland, aber auch für eine Reihe weiterer westeuropäischer Staaten, hohe Zusammenhangswerte zwischen den mit Wordscores geschätzten Parteipositionen und manuell erhobenen ideologischen Positionswerten im Zeitverlauf (vgl. Bräuninger et al. 2013), sodass von einer hohen Zuverlässigkeit der hier ermittelten Werte ausgegangen werden kann. ${ }^{1}$ Die Methode erlaubt auch die Messung der Positionen sowie der politikfeldspezifischen Salienzen der Parteien auf bzw. zu anderen Politikbereichen wie der Umwelt- oder Einwanderungspolitik. Da allerdings die Positionen deutscher Parteien zur Umweltpolitik stark mit wirtschaftsund gesellschaftspolitischen Fragen korrelieren und es einen hohen Zusammenhang zwischen der programmatischen Ausrichtung in gesellschafts- und migrationspolitischen Fragen gibt (vgl. Benoit und Laver 2006, S. 115, 118; Bräuninger et al. 2019b, S. 106-107), konzentriert sich der vorliegende Beitrag auf die Dimensio-

\footnotetext{
${ }^{1}$ Das Verfahren geht von der Beobachtung aus, dass die Wortwahl politischer Akteure in ihren programmatischen Dokumenten oder Reden nicht nach dem Zufallsprinzip erfolgt. Vielmehr wird angenommen, dass man allein aufgrund der relativen Worthäufigkeit eines Dokuments auf dessen programmatische Position schließen kann. Vorab werden Referenztexte identifiziert, denen für die jeweiligen Politikdimensionen Referenzwerte zugewiesen werden. Für eine detaillierte Beschreibung des Verfahrens und seiner Anwendung zur Ermittlung der Positionen der Landesparteien in Deutschland für den Zeitraum seit 1990 siehe Bräuninger et al. (2020, S. 52-68).
} 
nen Wirtschafts-, Sozial- und Finanzpolitik einerseits sowie Gesellschaftspolitik andererseits. ${ }^{2}$

Einem auf der Grundlage des Wordscores-Verfahrens bereits erstellten Datensatz, der die Positionen der deutschen Bundes- und Landesparteien seit Januar 1990 umfasst (Bräuninger et al. 2020), wurden die zu den Bundestagswahlen 2021 verfassten Wahlprogramme von CDU/CSU, SPD, AfD, FDP, Bündnis 90/Die Grünen und Die Linke sowie das separate Wahlprogramm der CSU hinzugefügt. Zudem erlaubt dieser Datensatz mithilfe bedingter logistischer Regressionen die Ermittlung der Determinanten der Koalitionsbildung in Bund und Ländern, auf deren Grundlage sich wiederum die jeweilige Wahrscheinlichkeit für jede mögliche Koalitionsoption ermitteln lässt, die sich aus der Anzahl der parlamentarisch vertretenen Parteien ergibt (Bräuninger und Debus 2008, S. 328-330; Linhart et al. 2010, S. 248-250; Debus und Müller 2011; Debus 2017; Bräuninger et al. 2019a).

Die zur Erklärung der Regierungs- und Koalitionsbildung herangezogenen Variablen können jedoch in einem engen Zusammenhang stehen. So sollte es wahrscheinlicher sein, dass Parteien, die eine wechselseitige Koalitionsaussage getätigt und somit signalisiert haben, gerne nach der Wahl in einer Regierung zusammenzuarbeiten, auch große Übereinstimmungen in ihren programmatischen Ausrichtungen haben, während die Distanz zu einer Partei, die von einer oder mehreren Parteien als Koalitionspartner ausgeschlossen wird, größer ausfallen dürfte. Daher werden dem eigentlichen Conditional-Logit-Schätzmodell, welches die Determinanten der Regierungs- und Koalitionsbildung in Deutschland in Bund und Ländern seit 1990 ermittelt, zwei einfache Logit-Modelle vorangestellt, in denen jeweils die Variablen „positive Koalitionsaussage“ und ,abgelehnte Koalition“ die abhängigen Variablen darstellen und alle anderen theoretisch hergeleiteten Faktoren die unabhängigen Variablen bilden. Die sich aus diesen beiden Logit-Regressionen ergebenden Residuen und damit der nicht durch die anderen Determinanten der Koalitionsbildung erklärte Anteil der beiden Variablen ,,positive Koalitionsaussage“ und „,abgelehnte Koalition“ werden - um deren Verwobenheit mit den anderen Erklärungsfaktoren der Regierungs- und Koalitionsbildung Rechnung zu tragen - als erklärende Faktoren in die bedingte logistische Regression integriert, die die Einflussgrößen aller unabhängigen Variablen auf den Ausgang des Regierungs- und Koalitionsbildungsprozesses bestimmt (vgl. Bräuninger und Debus 2008, S. 331-333; Bräuninger et al. 2020, S. 223). Bevor die Ergebnisse der bedingten logistischen Regression und auf dieser Grundlage die Wahrscheinlichkeiten verschiedener Koalitionsoptionen in unterschiedlichen Szenarien erläutert werden, präsentiert der folgende Abschnitt Infor-

\footnotetext{
2 Im Hinblick auf das Heranziehen von Wahlprogrammen zur Messung der programmatischen Positionen der Parteien ist kritisch anzumerken, dass Wahlprogramme selbst von den Koalitionsstrategien der Parteien beeinflusst werden können, etwa um nach der Wahl in eine optimale Verhandlungssituation zu gelangen (Debus 2005). Allerdings - und dies hat die Bundestagswahl 2021 eindrücklich gezeigt - können die zum Zeitpunkt des Verfassens der Wahlprogramme - also in der Regel mehrere Monate vor der Wahl wahrscheinlich mit einer Mehrheit ausgestatteten Koalitionen sich massiv von den Mehrheitskoalitionen unterscheiden, die sich aufgrund des Wahlergebnisses ergeben. Insofern sollten - bei einem Elektorat, dass sich zu einem anwachsenden Anteil spät im Wahlkampf für eine Partei entscheidet (Schmitt-Beck und Partheymüller 2012) - koalitionsstrategische Positionierungen in Wahlprogrammen geringer ausfallen als zu früheren Wahlen.
} 
mationen zum Policy-Profil der im Bundestag vertretenen Parteien auf der Grundlage der Wordscores-Inhaltsanalyse.

\section{Programmatisches Profil der Parteien zur Bundestagswahl 2021}

Die im Bundestag vertretenen Parteien haben - im Vergleich zu $2017^{3}$ - ihre Positionen teils deutlich verschoben. So ist das Wahlprogramm der Linken wirtschafts- wie gesellschaftspolitisch noch staatsinterventionistischer bzw. progressiver ausgerichtet. Auch die AfD nimmt extremere Positionen ein: So ist ihr Wahlprogramm 2021 marktliberaler und gesellschaftspolitisch deutlich traditionell-konservativer ausgerichtet als noch 2017. Da insbesondere die AfD, aber auch - zumindest von Union und FDP, aber auch von nicht unbedeutenden Teilen der SPD und der Grünen - die Linke auf Bundesebene als nicht koalitionsfähig erachtet werden, sind die Positionsverschiebungen von Christdemokraten, Sozialdemokraten, Liberalen und Grünen von besonderem Interesse im Hinblick auf die Realisierbarkeit möglicher Koalitionsoptionen. Zentral ist hier die nunmehr gesellschaftspolitisch zentristische Position der CDU/CSU, die 2017 noch moderat-traditionelle Positionen vertreten hat. Selbst die CSU, die ein separates Wahlprogramm zusätzlich zum gemeinsamen Dokument mit der CDU vorgelegt hat, nimmt weniger traditionelle Positionen in Fragen der Gesellschaftspolitik ein als die Union in ihrem 2017er-Wahlprogramm. Gleichzeitig ist die CSU zentristischer in wirtschafts- und sozialpolitischen Fragen ausgerichtet als die Union insgesamt, selbst wenn man den statistischen Schwankungsbereich der

Abb. 1 Programmatische Positionen der im Bundestag vertretenen Parteien, 2017-2021. (Quelle: Fortschreibung des Datensatzes von Bräuninger et al. 2020)

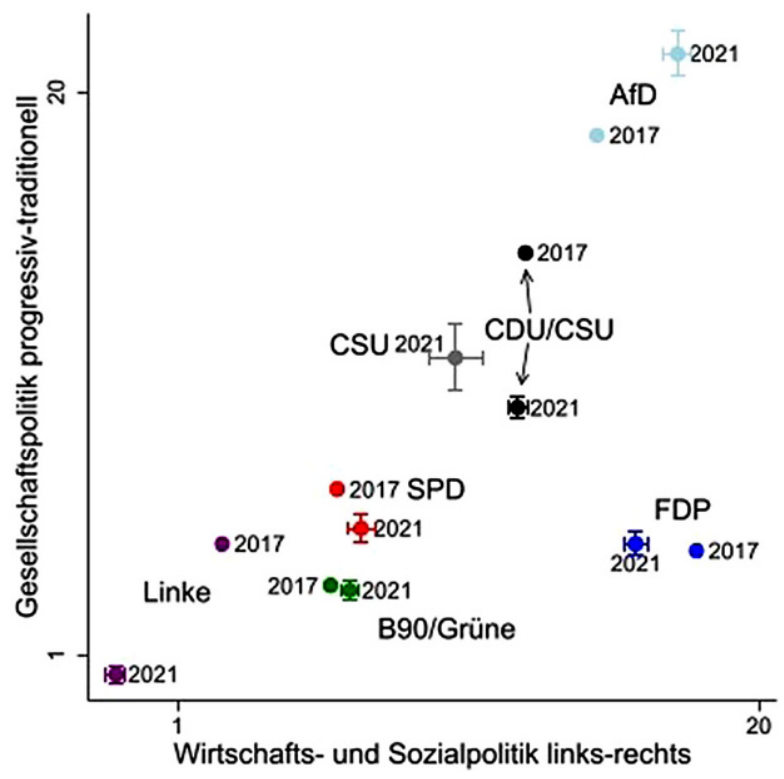

3 Die Positionen der Bundesparteien 2017 entsprechen den Ergebnissen der Expertenbefragung von Bräuninger et al. (2019b), die auch als Referenzwerte in die Wordscores-Schätzung mit eingehen (für eine detaillierte Schilderung der methodischen Vorgehensweise siehe Bräuninger et al. 2020, S. 59-67). 
Tab. 1 Parteispezifische relative Bedeutung (Salienz) der Politikfelder Wirtschaft und Soziales sowie Gesellschaft

\begin{tabular}{lll}
\hline & $\begin{array}{l}\text { Relative Salienz Wirtschafts- und Sozialpo- } \\
\text { litik }\end{array}$ & $\begin{array}{l}\text { Relative Salienz Gesellschaftspoli- } \\
\text { tik }\end{array}$ \\
\hline CDU/CSU & 0,562 & 0,438 \\
SPD & 0,523 & 0,477 \\
AfD & 0,277 & 0,723 \\
FDP & 0,621 & 0,379 \\
Bündnis 90/ & 0,458 & 0,542 \\
Grüne & & 0,480 \\
Linke & 0,520 & \\
\hline
\end{tabular}

Quelle: Fortschreibung des Datensatzes von Bräuninger et al. (2020)

sich aus der Wordscores-Schätzung ergebenden Position berücksichtigt. Während die Freien Demokraten ihre sehr marktliberale Position im Vergleich zu 2017 etwas abschwächten, haben sich die wirtschafts- und sozialpolitischen Positionen von SPD und Grünen kaum verändert. Vor dem Hintergrund, dass die programmatischen Positionen der Parteien einen zentralen Erklärungsfaktor für die Koalitionsbildung bilden und solche Koalitionen wahrscheinlicher sind, deren programmatische Heterogenität geringer ausfällt, sollte - im Vergleich zu 2017 - eine Kompromissfindung zwischen CDU/CSU, Grünen und FDP vor allem aufgrund der Positionsverschiebung der Union deutlich leichter fallen. Auch die - wenn auch geringer ausfallenden - Änderungen in den wirtschafts- und sozialpolitischen Positionen von Liberalen, Grünen und SPD sollten die Möglichkeiten auf Bildung einer „Ampelkoalition“ aus den drei Parteien leicht anwachsen lassen, wohingegen ein Bündnis aus Grünen, SPD und Linken aufgrund der starken Verschiebung der Position der Linken an die Extreme der beiden hier berücksichtigten Politikdimensionen eher unwahrscheinlicher sein sollte (Abb. 1).

Diese über die Parteipositionen identifizierbaren Muster verschieben sich leicht, wenn man die Bedeutung oder Salienz berücksichtigt, die die beiden hier herangezogenen Politikfelder für die Parteien haben (Tab. 1). So nimmt etwa für die FDP die Wirtschafts- und Sozialpolitik im Vergleich zur Gesellschaftspolitik eine deutlich größere Bedeutung ein, was die programmatische Distanz zu den anderen Parteien in sozioökonomischen Fragen aus Sicht der FDP anwachsen lassen sollte. Dies verringert die Kompromissbereitschaft der Freien Demokraten in dem für sie wichtigen Politikfeld Wirtschaft, Finanzen und Soziales insofern, dass Parteien wie Grüne oder SPD, die tendenziell staatsinterventionistische Positionen in diesem Politikfeld einnehmen, aus Sicht der FDP noch weiter von der Position der Freien Demokraten entfernt liegen. Dies führte - in Kombination mit weiteren Faktoren bereits 2017 zu einem vorzeitigen Ende der Verhandlungen über eine ,Jamaika-Koalition“ (Bräuninger et al. 2019a; Faas und Klingelhöfer 2019). Dass für die Grünen die Gesellschaftspolitik von größerer Bedeutung als die Wirtschafts- und Sozialpolitik ist, mag wiederum die Kompromissfindung mit der CDU/CSU trotz deren im Vergleich zu 2017 deutlich moderateren Position erschweren, gleichzeitig aber eine Grundlage für eine Ampelkoalition bilden, die der FDP-Vorsitzende Christian Lindner nach der Wahl als ,fortschrittliches Zentrum“ beschrieb und so indirekt auf die 
großen Schnittmengen von FDP, Grünen und SPD in Fragen der Gesellschaftspolitik hinwies.

\section{Determinanten der Koalitionsbildung in Deutschland und Wahrscheinlichkeiten verschiedener Koalitionsoptionen nach der Bundestagswahl 2021}

Ein ausschließlicher Fokus auf die programmatische Ausrichtung der Parteien und ihre Schnittmengen mit ihren Mitbewerbern auf den zentralen, den deutschen Parteienwettbewerb strukturierenden Politikdimensionen ist jedoch nur eine Komponente, die dazu beiträgt, Regierungs- und Koalitionsbildung zu erklären. Vielmehr wird die parteipolitische Zusammensetzung einer Regierung auch vom Ziel der Parteien an der Besetzung möglichst vieler Kabinettsposten sowie von den institutionellkontextuellen Faktoren eines politischen Systems beeinflusst. Dies zeigen die Ergebnisse der bedingten logistischen Regression, in die alle Regierungs- und Koalitionsbildungsprozesse in Deutschland auf Bundes- und Landesebene seit Januar 1990 einfließen und die die skizzierten theoretischen Ansätze aus der office- und policy-orientierten Koalitionstheorie sowie institutionell-kontextuelle Merkmale berücksichtigt, von denen ein Einfluss auf den Regierungsbildungsprozess erwartet werden kann. ${ }^{4}$ Abb. 2 weist die Ergebnisse des Conditional-Logit-Modells aus. So sind in Deutschland solche Koalitionen signifikant wahrscheinlicher,

- die hinsichtlich ihres Policy-Profils, das die programmatischen Positionen als auch parteispezifischen Salienzen der Politikbereiche abdeckt, homogener in wirtschafts- und sozialpolitischen sowie in gesellschaftspolitischen Fragen sind;

- bei der jeder Koalitionspartner möglichst viele Ämter im Kabinett besetzen kann;

- die aus möglichst wenigen Parteien bestehen;

- die die stärkste Fraktion im Parlament umfassen, welche als Agendasetzer bei den Koalitionsverhandlungen agieren kann;

- die mit den vor der Wahl geäußerten, positiven wie negativen Koalitionssignalen übereinstimmen;

- die die amtierende Regierung stellen und

- die mit den Mustern der parteipolitischen Mehrheitssituation in der jeweils anderen Parlamentskammer übereinstimmen (vgl. auch Bräuninger und Debus 2008; Bräuninger et al. 2019a, 2020; Debus und Gross 2016).

Mithilfe der geschätzten Effektstärken werden im Folgenden die Wahrscheinlichkeiten dafür ermittelt, dass sich die theoretisch möglichen Koalitionen auch tatsächlich bilden. Um die Effekte unterschiedlicher Mehrheitssituationen sowie positiv wie negativ formulierter Koalitionsaussagen, die die Anzahl möglicher Koalitionen stark beeinflussen, zu ermitteln, werden sechs Szenarien durchgespielt. In allen Szenarien besteht der Bundestag aus sechs Fraktionen, sodass die Anzahl der theoretisch

\footnotetext{
${ }^{4}$ Die Koalitionsbildungsprozesse in den Bundesländern Berlin und Mecklenburg-Vorpommern 2021 gehen nicht in die Analyse ein.
} 


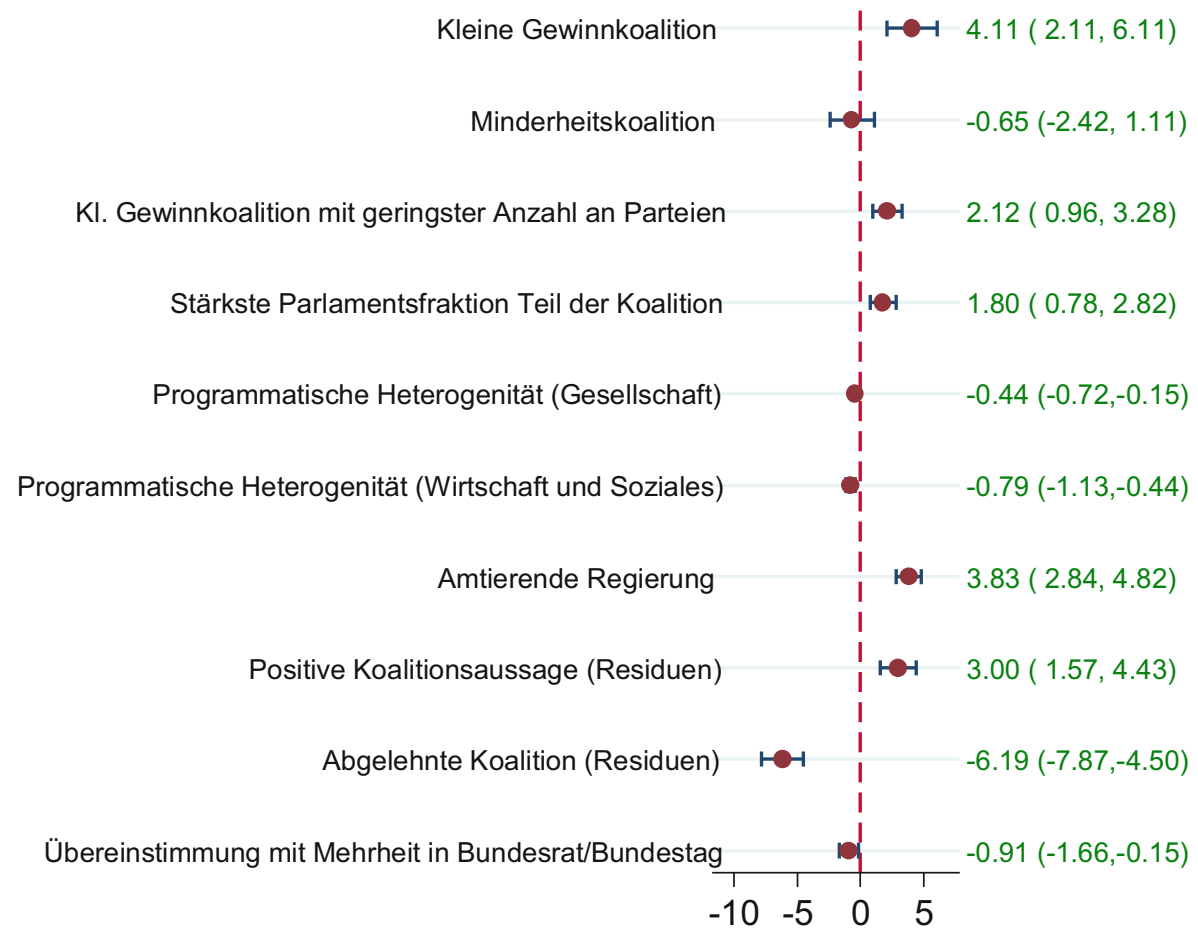

Abb. 2 Determinanten der Regierungs- und Koalitionsbildung in Deutschland auf Bundes- und Landesebene seit 1990. (Berechnungen auf der Grundlage der Fortschreibung des Datensatzes von Bräuninger et al. (2020). Balken geben das 90\%-Konfidenzintervall an. $N=3343$, Log Pseudolikelihood= $-73,37$, Pseudo $\mathrm{R}^{2}=0,81$. Anteil korrekt vorhergesagter Regierungs- und Koalitionsbildungen: $76 \%$ (95 von 125 Fälle))

möglichen Koalitionen damit - gemäß der Formal $2^{n}-1$, wobei $n$ für die Anzahl aller im Parlament vertretenen Parteien steht - bei 63 liegt.

Die ersten beiden Szenarien basieren auf dem Wahlergebnis vom 26.09.2021 und der entsprechenden Sitzverteilung, wobei im zweiten Szenario die Information eingebaut wird, dass eine Koalition unter Einschluss von Union und SPD vonseiten der jeweiligen Parteien zunächst ausgeschlossen wird. So sagte etwa SPD-Generalsekretär Klingbeil nach der Wahl, dass er eine erneute „große Koalition“ ablehne und sie auch kein Plan B im Falle eines Scheiterns der Verhandlungen über eine „Ampelkoalition" sei. ${ }^{5}$ Im dritten und vierten Szenario wird die Sitzverteilung so verändert, dass nicht die SPD, sondern CDU/CSU die stärkste Fraktion im Bundestag stellen, wobei in Szenario 4 wiederum eine Koalition, in denen Christ- und Sozialdemokraten vertreten wären, von den beiden Parteien ausgeschlossen wird, auch vor dem Hintergrund, dass der SPD-Vorsitzende Walter-Borjans vor der Wahl betonte, dass

\footnotetext{
5 Siehe https://www.deutschlandfunk.de/nach-der-bundestagswahl-2021-welche-koalitionen-sind-denk bar.2897.de.html?dram:article_id=502404\#deutschland (Zugriff am 13.10.2021).
} 


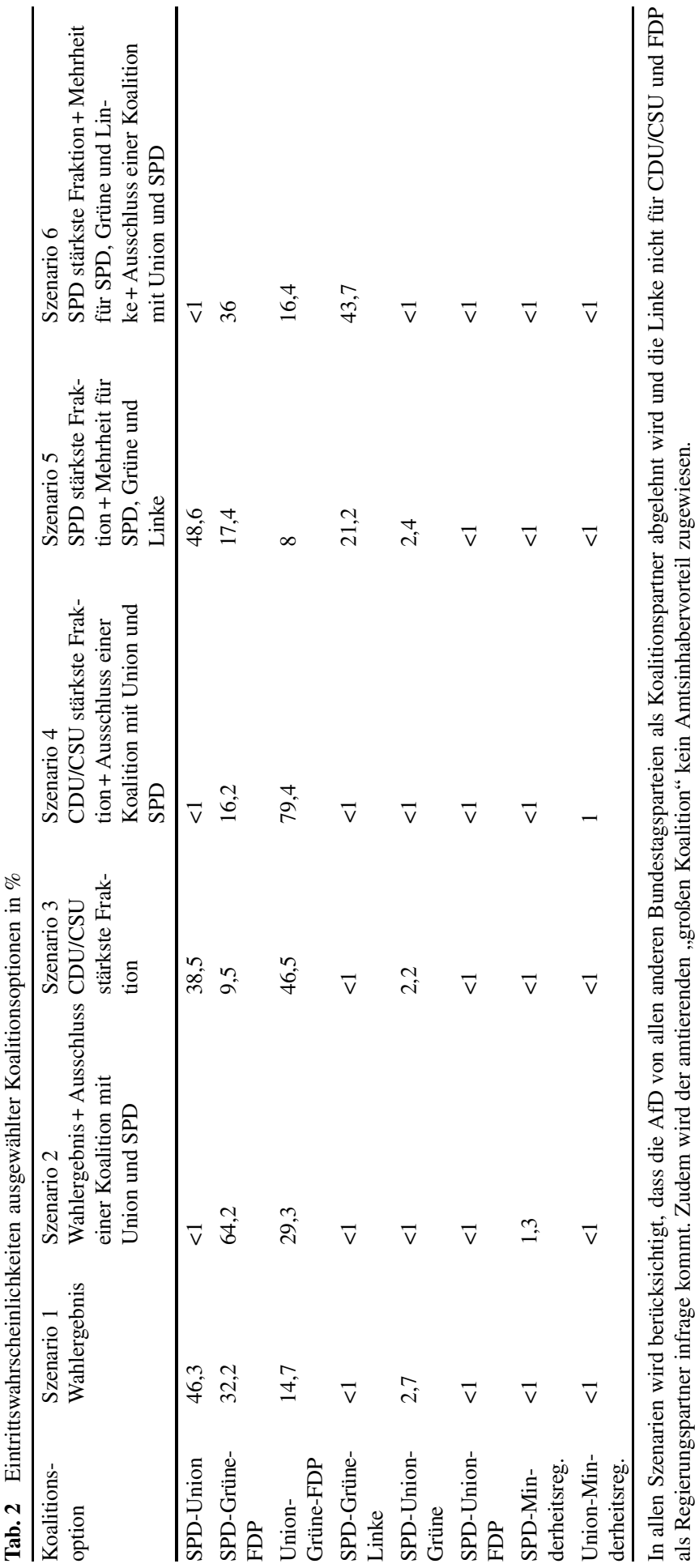


die SPD generell nur als stärkste Partei, die den Kanzler stelle, in eine Koalition eintreten würde. ${ }^{6}$ Das fünfte und sechste Szenario spiegelt jeweils einen Bundestag wider, in dem die SPD stärkste Fraktion ist und Sozialdemokraten, Grüne und Linke über eine Mehrheit der Sitze verfügen (was im 2021 gewählten Bundestag so nicht der Fall ist). In Szenario sechs wird wiederum die Information eingebaut, dass eine Koalition, die Christ- und Sozialdemokraten miteinschließt, abgelehnt wird. In allen sechs Szenarien wird der amtierenden Regierungskoalition aus CDU/CSU und SPD kein Amtsinhaberbonus zugewiesen. Zudem bestehen in allen sechs Szenarien Mehrheiten für eine „Ampel-“ und „Jamaika-Koalition“ und es wird berücksichtigt, dass die AfD von allen anderen Bundestagsparteien als Koalitionspartner abgelehnt wird sowie die Linke nicht für CDU/CSU und FDP als Regierungspartner infrage kommt.

Tab. 2 weist die Eintrittswahrscheinlichkeiten ausgewählter Koalitionsoptionen für die sechs dargelegten Szenarien aus. Ohne Berücksichtigung der spezifischen Koalitionspräferenzen von Union und Sozialdemokraten, nach der Wahl die Zusammenarbeit beider Fraktionen in einer Koalition nicht fortzusetzen, wäre eine Regierung aus SPD und CDU/CSU mit den Sozialdemokraten als stärkster Fraktion, die den Kanzler stellen würde, das wahrscheinlichste Ergebnis (Wahrscheinlichkeit: 46,3\%; Tab. 2, Szenario 1). Bezieht man jedoch ein, dass Vertreter von Union und SPD vor wie nach der Wahl geäußert haben, die ,große Koalition“ nicht fortzusetzen, ist die „Ampelkoalition“ aus SPD, Grünen und FDP das klar wahrscheinlichere Ergebnis des Regierungsbildungsprozesses 2021 und hätte eine Chance von 64,2\% auf Übernahme der Regierungsgeschäfte, wohingegen die Chancen für eine „Jamaika-Koalition“, bei der die SPD als stärkste Fraktion außen vor gelassen würde, bei nur gut $29 \%$ liegen (Tab. 2, Szenario 2).

Wäre nicht die SPD, sondern die CDU/CSU stärkste Fraktion geworden, dann wäre laut Szenario 3 in Tab. 2 die Bildung einer Koalition aus Union, Grünen und FDP wahrscheinlicher (46,5\%) gewesen als die erneute Bildung einer ,großen Koalition“ (38,5\%). Die Chance auf Bildung einer „Ampelkoalition“ - dann ohne die Union als stärkste Fraktion - hätte bei nur knapp $10 \%$ gelegen. Die Option „Jamaika“ dominiert klar das Bild mit einer Wahrscheinlichkeit auf Bildung von $79,4 \%$, wenn eine Koalition mit Union und SPD von den beiden Fraktionen als ausgeschlossen betrachtet wird (Szenario 4 in Tab. 2).

Hätte die Linke bei der Wahl besser abgeschnitten - etwa zulasten der AfD und hätte es eine Mehrheit für eine ,rot-grün-rote“ Koalition gegeben, dann wäre diese Option das knapp wahrscheinlichste Resultat des Regierungsbildungsprozesses gewesen $(43,7 \%)$, wenn man eine Koalition mit SPD und Union als ausgeschlossen betrachtet (Szenario 6 in Tab. 2). Ignoriert man die Statements von Union und SPD vor und nach der Wahl, nicht miteinander koalieren zu wollen, dann wäre - auch bei einer parlamentarischen Mehrheit für eine Koalition aus SPD, Grünen und Linken - die Fortsetzung der ,großen“ Koalition wahrscheinlicher gewesen (48,6\%; Szenario 5 in Tab. 2).

\footnotetext{
6 Siehe https://www.rnd.de/politik/fuehren-oder-nichts-walter-borjans-will-spd-nach-bundestagswahlnicht-als-juniorpartner-EH7CPZ4LZCRBW46E22HNXAGN54.html (Zugriff am 13.10.2021).
} 
Zusammengefasst variiert - je nach Sitzverteilung im Bundestag und der Frage, welche Fraktion am stärksten wird - deutlich, welches Ergebnis des Regierungsbildungsprozesses das wahrscheinlichste nach dem 26.09.2021 sein wird. Bezieht man den Unwillen von SPD und CDU/CSU ein, erneut eine ,große Koalition“ zu bilden - auch wenn dies nicht offiziell von Parteitagen so bestätigt wurde -, dann ist eine Koalition aus SPD, Grünen und FDP das wahrscheinlichste Ergebnis des Regierungsbildungsprozesses, wenn man die tatsächliche Sitzverteilung im 20. Deutschen Bundestag zugrunde legt. Wären die Unionsparteien hingegen stärkste Fraktion geworden, dann hätte viel für eine „Jamaika-Koalition“ gesprochen, wohingegen für den Fall einer ,rot-grün-roten“ Mehrheit im Bundestag eine Realisierung einer solchen Koalition wahrscheinlicher gewesen wäre als die Bildung einer ,Ampelkoalition“. Wird die Ablehnung einer ,großen Koalition“ durch Union und SPD außer Acht gelassen (wie in Szenarien 1, 3 und 5), dann ist diese Parteienkombination das wahrscheinlichste Resultat des Regierungsbildungsprozesses im neu gewählten Bundestag wie auch in einem Bundestag mit einer ,,rot-grün-roten“ Mehrheit. Lediglich in einem Bundestag mit CDU/CSU als stärkster Fraktion hätte - dem hier herangezogenen Modell zufolge - auch bei einer Berücksichtigung der ,großen Koalition“ aus Union und SPD eine „Jamaika-Koalition“ die höhere Wahrscheinlichkeit auf Bildung gehabt.

\section{Schlussbetrachtung und Ausblick}

Das fluider werdende bundesdeutsche Parteiensystem lässt althergebrachte Koalitionsoptionen wie Bündnisse aus CDU/CSU und FDP oder aus SPD und Bündnis 90/Die Grünen unwahrscheinlicher werden, auch wenn sie nach wie vor möglich sind. So konnte Armin Laschet 2017 in Nordrhein-Westfalen trotz des Einzugs der AfD in den Düsseldorfer Landtag seine Wunschkoalition mit der FDP bilden; nach der Landtagswahl in Baden-Württemberg 2021 fehlten nur wenige Stimmen für ein weiteres Mandat für Grüne oder SPD, was einem ,grün-roten“ Bündnis im Stuttgarter Landtag die absolute Mehrheit gebracht hätte. Die Bundestagswahl vom 26.09.2021 hat das Ergebnis erbracht, dass - wenn eine erneute ,große Koalition" vermieden werden soll - eine ideologisch eher heterogene Koalition aus drei Fraktionen gebildet werden muss. Ziel dieses Beitrags war zum einen die Präsentation der Ausprägungen des programmatischen Parteienwettbewerbs in Deutschland 2021, die auf der Grundlage einer Wordscores-Inhaltsanalyse der Wahlprogramme ermittelt wurden. Insbesondere die Union verschob in gesellschaftspolitischen Fragen ihre Position in Richtung von SPD, FDP und Grünen, sodass ein Bündnis aus CDU/CSU und einem oder mehreren der drei anderen Parteien leichter zu bilden sein sollte. Gleichzeitig bestehen nach wie vor deutliche Unterschiede zwischen den Parteien insbesondere in der Wirtschafts-, Sozial- und Finanzpolitik, die Koalitionen erschweren sollten, die FDP, Grüne, SPD oder auch Die Linke miteinbeziehen. Unter Berücksichtigung der weiteren relevanten Determinanten der Koalitionsbildung, die sich aus der theoretischen Literatur ableiten lassen, wurde deutlich, dass gerade unter Einbeziehung der Koalitionspräferenzen und -signale der Parteien bzw. 
ihrer zentralen Repräsentanten im gewählten Bundestag eine „Ampelkoalition“ das wahrscheinlichste Ergebnis des Regierungsbildungsprozesses 2021 ist.

Natürlich kann das hier präsentierte Schätzmodell nicht alle relevanten Faktoren, welche die Koalitionsbildung beeinflussen, in die Analyse einbauen. So berücksichtigen Parteien bei der Regierungsbildung durchaus die Koalitionswünsche der Wählerschaft und versuchen - aus Angst vor Abstrafung bei den folgenden Wahlen diese auch umzusetzen (Debus und Müller 2013). Eine von einer Mehrheit der Wählerinnen und Wähler nicht präferierte Koalition aus Union, Grünen und FDP sollte die drei Parteien davor zurückschrecken lassen, eine solche Koalition zu bilden, da sie bei folgenden (Landtags-)Wahlen aufgrund der Bildung einer solchen unpopulären Koalition, die dem Wahlverlierer CDU/CSU das Kanzleramt bringen würde, an Unterstützung einbüßen könnten. Eine weitere Perspektive der Koalitionsbildung, die nicht im Prognosemodell berücksichtigt werden konnte, bezieht die innerparteiliche Zerstrittenheit der Parteien mit ein (vgl. Luebbert 1986; Bäck 2008). Parteien, die durch große innerparteiliche Konflikte gekennzeichnet sind, dürften eher unattraktive Koalitionspartner für die anderen Parteien sein, da sie sich inhaltlich besser durchsetzen können (Baumann et al. 2017). Zudem ist im Fall der CDU aufgrund ihres schlechten Wahlergebnisses unklar, wer die Parteiführung übernehmen soll und dementsprechend wie sich die Christdemokraten inhaltlich aufstellen werden, was ebenfalls eine Hürde für die Bildung einer Koalition ist, der die CDU angehört.

Sollte sich eine Koalition aus SPD, Grünen und FDP bilden, dann wird es für die Koalitionsforschung von großem Interesse sein zu evaluieren, wie sich das Regieren in einer programmatisch durchaus komplexen Regierungskoalition ausgestaltet, sowohl hinsichtlich der Ämterverteilung und inhaltlichen Kompromissfindung im Koalitionsabkommen als auch im täglichen Prozess des Handelns und Entscheidens während der Legislaturperiode.

Funding Open Access funding enabled and organized by Projekt DEAL.

Open Access Dieser Artikel wird unter der Creative Commons Namensnennung 4.0 International Lizenz veröffentlicht, welche die Nutzung, Vervielfältigung, Bearbeitung, Verbreitung und Wiedergabe in jeglichem Medium und Format erlaubt, sofern Sie den/die ursprünglichen Autor(en) und die Quelle ordnungsgemäß nennen, einen Link zur Creative Commons Lizenz beifügen und angeben, ob Änderungen vorgenommen wurden.

Die in diesem Artikel enthaltenen Bilder und sonstiges Drittmaterial unterliegen ebenfalls der genannten Creative Commons Lizenz, sofern sich aus der Abbildungslegende nichts anderes ergibt. Sofern das betreffende Material nicht unter der genannten Creative Commons Lizenz steht und die betreffende Handlung nicht nach gesetzlichen Vorschriften erlaubt ist, ist für die oben aufgeführten Weiterverwendungen des Materials die Einwilligung des jeweiligen Rechteinhabers einzuholen.

Weitere Details zur Lizenz entnehmen Sie bitte der Lizenzinformation auf http://creativecommons.org/ licenses/by/4.0/deed.de.

Conflict of Interest M. Debus has no competing interests to declare that are relevant to the content of this article. 


\section{Literatur}

Axelrod, Robert M. 1970. Conflict of interest: a theory of divergent goals with applications to politics. Chicago: Markham Publishing Company.

Bäck, Hanna. 2008. Intra-party politics and coalition formation-Evidence from Swedish local government. Party Politics 14(1):71-89.

Bäck, Hanna, und Patrick Dumont. 2007. Combining large-n and small-n strategies: the way forward in coalition research. West European Politics 30(3):467-501.

Bäck, Hanna, und Patrick Dumont. 2008. Making the first move. A two-stage analysis of the role of formateurs in parliamentary government formation. Public Choice 135(3-4):353-373.

Bakker, Ryan, Catherine de Vries, Erica Edwards, Liesbet Hooghe, Seth Jolly, Gary Marks, Jonathan Polk, Jan Rovny, Marco Steenbergen, und Milada Anna Vachudova. 2015. Measuring party positions in Europe: The Chapel Hill expert survey trend file, 1999-2010. Party Politics 21(1):143-152.

Baumann, Markus, Marc Debus, und Martin Gross. 2017. Strength of weakness? Innerparteiliche Heterogenität, divergierende Koalitionspräferenzen und die Ergebnisse von Koalitionsverhandlungen in den deutschen Bundesländern. Politische Vierteljahresschrift 58(2):179-204.

Benoit, Kenneth, und Michael Laver. 2006. Party policy in modern democracies. London: Routledge.

Bergman, Torbjörn. 1993. Formation rules and minority governments. European Journal of Political Research 23(1):55-66.

Bräuninger, Thomas, und Marc Debus. 2008. Der Einfluss von Koalitionsaussagen, programmatischen Standpunkten und der Bundespolitik auf die Regierungsbildung in den deutschen Ländern. Politische Vierteljahresschrift 49(2):309-338.

Bräuninger, Thomas, Marc Debus, und Jochen Müller. 2013. Estimating policy positions of political actors across countries and time. MZES Arbeitspapier Nr. 153. Mannheim: Mannheimer Zentrum für Europäische Sozialforschung.

Bräuninger, Thomas, Marc Debus, Jochen Müller, und Christian Stecker. 2019a. Party competition and government formation in Germany: business as usual or new patterns? German Politics 28(1):80-100.

Bräuninger, Thomas, Marc Debus, Jochen Müller, und Christian Stecker. 2019b. Die programmatischen Positionen der deutschen Parteien zur Bundestagswahl 2017: Ergebnisse einer Expertenbefragung. In Jahrbuch für Handlungs- und Entscheidungstheorie, Hrsg. M. Debus, M. Tepe, und J. Sauermann, 93-113. Wiesbaden: Springer VS.

Bräuninger, Thomas, Marc Debus, Jochen Müller, und Christian Stecker. 2020. Parteienwettbewerb in den deutschen Bundesländern. Wiesbaden: Springer VS.

Däubler, Thomas, und Marc Debus. 2009. Government formation and policy formulation in the German states. Regional and Federal Studies 19(1):73-95.

De Swaan, Abram. 1973. Coalition theories and cabinet formations: A study of formal theories of coalition formation applied to nine European parliaments after 1918. Amsterdam: Elsevier.

Debus, Marc. 2005. Annähern oder Distanzieren? Strategische Positionierung von FDP, VdU und FPÖ zwischen 1949 und 2002. Österreichische Zeitschrift für Politikwissenschaft 34(4):413-430.

Debus, Marc. 2009. Pre-electoral commitments and government formation. Public Choice 138(1-2):45-64.

Debus, Marc. 2017. Verrückte Verhältnisse? Wahlverhalten und Parteienwettbewerb in Baden-Württemberg zwischen 2011 und 2016. In Das grün-rote Experiment in Baden-Württemberg, Hrsg. F. Hörisch, S. Wurster, 15-45. Wiesbaden: Springer VS.

Debus, Marc, und Martin Gross. 2016. Coalition formation at the local level: institutional constraints, party policy conflict, and office-seeking political parties. Party Politics 22(6):835-846.

Debus, Marc, und Jochen Müller. 2011. Government formation after the 2009 federal election: the remake of the Christian-liberal coalition under new patterns of party competition. German Politics 20(1):164-185.

Debus, Marc, und Jochen Müller. 2013. Do voters' coalition preferences affect government formation? West European Politics 36(5):1007-1028.

Debus, Marc, Holger Döring, und Alejandro Ecker. 2021. Germany: from stable coalition camps to new complexity. In Coalition governments in western Europe, Hrsg. T. Bergman, H. Bäck, und J. Hellström, 247-283. Oxford: Oxford University Press.

Faas, Thorsten, und Tristan Klingelhöfer. 2019. The more things change, the more they stay the same? The German federal election of 2017 and its consequences. West European Politics 42(4):914-926.

Golder, Sona Nadenichek. 2006. Pre-electoral coalition formation in parliamentary democracies. British Journal of Political Science 36(2):193-212. 
Jankowski, Michael, Christian Stecker, Andreas Blätte, Thomas Bräuninger, Marc Debus, Anna-Sophie Kurella, Jochen Müller, und Susanne Pickel. 2021. Open expert survey 2021. https://doi.org/10.7910/ DVN/XIL6OW. Harvard Dataverse, V1.

Kropp, Sabine. 2001. Regieren in Koalitionen: Handlungsmuster und Entscheidungsbildung in deutschen Länderregierungen. Wiesbaden: Westdeutscher Verlag.

Laver, Michael, und Norman Schofield. 1998. Multiparty government: the politics of coalition in europe. Ann Arbor: University of Michigan Press.

Laver, Michael, und Kenneth A. Shepsle. 1996. Making and breaking governments: cabinets and legislatures in parliamentary democracies. Cambridge: Cambridge University Press.

Laver, Michael, Kenneth Benoit, und John Garry. 2003. Extracting policy positions from political texts using words as data. American Political Science Review 97(2):311-331.

Linhart, Eric, Marc Debus, und Thomas Bräuninger. 2010. The 2009 elections in Schleswig-Holstein: polarised electoral campaign, exceptional election results, and an unspectacular process of government formation. German Politics 19(2):237-253.

Luebbert, Gregory M. 1986. Comparative democracy: policymaking and governing coalitions in Europe and Israel. New York: Columbia University Press.

Martin, Lanny W., und Randolph T. Stevenson. 2001. Government formation in parliamentary democracies. American Journal of Political Science 45(1):33-50.

Martin, Lanny W., und Randolph T. Stevenson. 2010. The conditional impact of incumbency on government formation. American Political Science Review 104(3):503-518.

Pappi, Franz Urban, und Susumu Shikano. 2004. Ideologische Signale in den Wahlprogrammen der deutschen Bundestagsparteien 1980 bis 2002. MZES Arbeitspapier Nr. 76. Mannheim: Mannheimer Zentrum für Europäische Sozialforschung.

Pappi, Franz Urban, Axel Becker, und Alexander Herzog. 2005. Regierungsbildung in Mehrebenensystemen: Zur Erklärung der Koalitionsbildung in den deutschen Bundesländern. Politische Vierteljahresschrift 46(3):432-458.

Riker, William H. 1962. The theory of political coalitions. New Haven: Yale University Press.

Schmitt-Beck, Rüdiger, und Julia Partheymüller. 2012. Why voters decide late: a simultaneous test of old and new hypotheses at the 2005 and 2009 German federal elections. German Politics 21(3):299-316.

Stecker, Christian. 2016. The effects of federalism reform on the legislative process in Germany. Regional \& Federal Studies 26(5):603-624.

Stecker, Christian. 2017. Die „stabile Mehrheit“ ist völlig überschätzt. https://www.tagesspiegel.de/politik/ regierungsfindung-die-stabile-mehrheit-ist-voellig-ueberschaetzt/20677900.html. Zugegriffen: 6. Aug. 2021.

Strøm, Kaare, Ian Budge, und Michael Laver. 1994. Constraints on cabinet formation in parliamentary democracies. American Journal of Political Science 38(2):303-335.

Volkens, Andrea, Pola Lehmann, Theres Matthieß, Nicolas Merz, Sven Regel, und Bernhard Weßels. 2017. The Manifesto Data Collection. Manifesto Project (MRG/CMP/MARPOR). Version 2017b. Berlin: Wissenschaftszentrum Berlin für Sozialforschung (WZB). https://doi.org/10.25522/manifesto.mpds. $2017 b$

von Neumann, Jon, und Oskar Morgenstern. 1944. Theory of games and economic behavior. Princeton: Princeton University Press. 